\title{
Serum Lipids are Novel Predictors for Thyroid Autoimmunity in the General Population with Normal TSH Levels From A Cross-Sectional Study
}

\section{Jiarong Li}

The First Hospital of China Medical University: The First Affiliated Hospital of China Medical University

\section{Zixiao Wang}

The First Hospital of China Medical University: The First Affiliated Hospital of China Medical University He Liu

The First Hospital of China Medical University: The First Affiliated Hospital of China Medical University Jinrong Fu

The First Hospital of China Medical University: The First Affiliated Hospital of China Medical University

\section{Fengye Qin}

The First Hospital of China Medical University: The First Affiliated Hospital of China Medical University Haixia Guan ( $\nabla$ hxguan@vip.126.com )

Guangdong Provincial People's Hospital https://orcid.org/0000-0002-4465-1441

\section{Wei Wang}

The First Hospital of China Medical University: The First Affiliated Hospital of China Medical University

\section{Research Article}

Keywords: Low-density lipoprotein cholesterol, High-density lipoprotein cholesterol, Thyroglobulin antibody, Thyroid peroxidase antibody, Thyroid, Autoimmunity

Posted Date: February 18th, 2021

DOl: https://doi.org/10.21203/rs.3.rs-185316/v1

License: (a) (1) This work is licensed under a Creative Commons Attribution 4.0 International License. Read Full License 


\section{Abstract \\ Objective}

Serum lipids have been shown to regulate inflammatory and immune processes, but little is known about their association with thyroid autoimmunity. This study aimed to investigate the association of serum lipids with thyroid autoantibody positivity in the general population with normal thyroid-stimulating hormone (TSH) levels.

\section{Methods}

Data regarding the 7,688 subjects' baseline characteristics were retrospectively collected. All subjects were categorized into four groups according to thyroid autoantibodies against thyroglobulin (TgAb) and thyroid peroxidase (TPOAb) positivity and serum lipid levels were compared. Binary logistic regression models were used to evaluate the risk of TgAb or TPOAb positivity with increasing serum lipid levels.

\section{Results}

In 6,456 included subjects, after adjusting for confounders, the risk of TgAb positivity was positively associated with increasing low-density lipoprotein cholesterol (LDL-C) levels (OR 1.10, 95\% Cl 1.02-1.19, $P=0.014$ ) and negatively correlated with the increasing high-density lipoprotein cholesterol (HDL-C) levels (OR $0.80,95 \% \mathrm{Cl} 0.67-0.96, P=0.014)$. In female subjects, the association between increasing LDLC (OR 1.15, 95\% Cl 1.05-1.26, $P=0.004)$ or HDL-C levels (OR 0.74, 95\% Cl 0.60-0.91, $P=0.005$ ) and TgAb positivity become more pronounced. Moreover, there was a positive correlation between LDL-C levels and TPOAb positivity (OR 1.14, 95\% Cl 1.02-1.26, $P=0.016)$.

\section{Conclusion}

We have shown the associations of HDL-C and LDL-C with thyroid autoantibody positivity in the general population with normal TSH levels in a gender-dependent manner. This study highlights that serum lipids may be new predictors of thyroid autoimmunity even when TSH is within the reference range.

\section{Introduction}

Thyroid autoantibodies against thyroglobulin (TgAb) and thyroid peroxidase (TPOAb) are key markers of thyroid autoimmunity. These antibodies are of great value for the diagnosis and management of autoimmune thyroid disease (AITD), which predominately consists of Hashimoto thyroiditis (HT or chronic lymphocytic thyroiditis). According to a 2020 epidemiological survey by our group [1], the overall prevalence of positive thyroid autoantibodies was $14.19 \%$ in the Chinese population. $\operatorname{TgAb}$ positive and TPOAb negative (TPOAb-TgA $b^{+}$) status along with TPOAb positive and TgAb negative (TPOA $\left.{ }^{+}{ }^{+} \mathrm{TgAb} \mathrm{b}^{-}\right)$ 
status were detected in $9.70 \%$ and $10.19 \%$, respectively. The prevalence of TgAb or TPOAb positivity $\left(\mathrm{TPOAb}^{+} / \mathrm{TgAb}{ }^{+}\right)$was significantly higher in women than in men and increased with age.

In the 1960s, the relationship between thyroid autoimmunity and hyperlipidemia was suggested [2], and dyslipidemia was conventionally thought to be a consequence of AITD. For example, Tamer et al. [3] indicated that thyroid autoimmunity may be a risk factor for hyperlipidemia independent of the thyroid function in patients with HT. On comparison with TPOAb-negative subjects, Mazaheri et al. [4] reported that hypothyroid patients with TPOAb levels $>1000 \mathrm{IU} / \mathrm{mL}$ may have lower high-density lipoprotein cholesterol (HDL-C) levels. This is further supported by Kang et al. [5] who concluded that high TPOAb levels above the normal range can lead to dyslipidemia in euthyroid population. In AITD patients, Chen et al. [6] showed that the risk of hyperlipidemia also increased even when thyroid-stimulating hormone (TSH) levels were within the normal range. Moreover, a prospective observational study conducted by Srivastava et al. demonstrated that dyslipidemia was found in $100 \%$ of subclinical hypothyroidism patients with positive TPOAb [7].

Alternatively, recent evidence emerges that serum lipids may be involved in the development of AITD [810]. Stein et al. [11] and Gullu et al. [12] contend that statins or 3-hydroxy-3-methylglutaryl coenzyme A (HMG-CoA) reductase inhibitors could produce anti-inflammatory and immune-suppressing effects at the level of the thyroid gland. Wu et al. [13] showed that simvastatin and atorvastatin were able to inhibit the human leukocyte antigen D-related (HLA-DR) expression on thyrocytes of patients with AITD. In addition, Krysiaket al. [14] affirmed that fenofibrate, a peroxisome proliferator-activated receptor-a (PPAR-a) activator (i.e., fibrates class of drugs), could decrease serum titers of TgAb and TPOAb in HT patients.

Regardless of the direction of causality between dyslipidemia and AITD, the inflammatory and immune pathways have central roles in the pathogenesis of both dyslipidemia and AITD. We submit that there may be common pathways between these two diseases through the action of thyroid autoantibodies TgAb and TPOAb. This study aimed at investigating the associations of increasing serum lipid levels with thyroid autoantibody positivity in the general population with normal TSH levels.

\section{Materials And Methods}

\section{Study participants and design}

A total of 7,688 individuals were enrolled in a study as part of a routine health check-up at the Health Medical Center of the First Hospital of China Medical University from January 2017 to December 2018. The inclusion criteria of the participants were: (1) aged 14 years or older and (2) received thyroid function and antithyroid antibodies testing. Exclusion criteria included: under 14 years old $(n=0)$, missing serum lipids ( $n=108)$, abnormal TSH levels $(n=459)$, had a history of thyroid surgery $(n=199)$ or incomplete data $(n=466)$. The database lacked information on medication (including thyroid medication and lipid mediation), smoking, alcohol consumption, and other concomitant autoimmune diseases. Finally, 6,456 subjects were included in this retrospective cross-sectional study. 


\section{Data Collection}

All data were retrospectively collected from the hospital information system. During the routine health check-up, weight and height were measured having participants wear light clothing and no shoes. Body mass index (BMI) was calculated as weight in kilograms divided by the square of height in meters. Waist circumference (WC) was measured at the umbilicus level in a standing position. Systolic blood pressure (SBP) were measured twice consecutively in the same arm at 2-minute interval between the measurements with the subjects in a seated position after at least 5 minutes of rest. The average of the two SBP measurements were used in subsequent statistical analysis.

\section{Biochemical measurements}

Venous blood samples were collected in the morning between 7:00 am to 10:00 am after an 8-12 hour fast, and then promptly centrifuged and analyzed. The concentrations of fasting plasma glucose (FPG), total cholesterol (TC), triglyceride (TG), low-density lipoprotein cholesterol (LDL-C), and HDL-C were determined by enzymatic methods on the Automatic Biochemical Analyzer.

\section{Thyroid indexes measurements}

The levels of serum TSH, TgAb, and TPOAb were determined in all subjects on the same day of blood sampling, using the automated immunochemiluminescent assay (ICMA) kits (Abbott, IL, USA). The reference ranges of TSH, TgAb, TPOAb were 0.35-4.94 $\mathrm{mlU} / \mathrm{L},<4.11 \mathrm{IU} / \mathrm{ml},<5.61 \mathrm{IU} / \mathrm{ml}$, respectively, and the intra- and inter-assay coefficient of variation for these serum parameters were $<8 \%$. TSH abnormality was defined as greater than $4.94 \mathrm{mIU} / \mathrm{L}$ or less than $0.35 \mathrm{mIU} / \mathrm{L}$ according to the ICMA kit. TgAb and TPOAb positivity were considered at $\geq 4.11 \mathrm{IU} / \mathrm{ml}$ and $\geq 5.61 \mathrm{IU} / \mathrm{ml}$, respectively.

\section{Statistical Analysis}

Continuous variables were shown as mean \pm standard deviation (SD) for those with normal distribution and were described as median (first quartile-third quartile) for those with skewed distribution. Oneway ANOVA and the Kruskal-Wallis test were used for intergroup comparisons. All categorical variables were presented as count (percentage) and the Pearson $\chi^{2}$ test was used to compare variables across different groups. The associations between serum lipid levels (continuous variable) and TgAb or TPOAb positivity (categorical variable) were assessed by binary logistic regression models. Model 1 adjusted for age and/or gender, and Model 2 further adjusted for BMI, WC, SBP and FPG. Results were expressed as odds ratios (OR) with $95 \%$ confidence interval $(\mathrm{Cl})$. All statistical analyses were performed with SPSS Statistics, Version 22.0 (IBM Corporation, NY, USA). A two-tailed P value of less than 0.05 was deemed to be statistically significant.

\section{Results}

\section{Baseline characteristics of subjects}


A total of 6,456 subjects $(3,298$ women and 3,158 men) with normal TSH levels were evaluated. The mean age was $47.64 \pm 11.17$ years. The overall thyroid antibody positivity was $29.8 \%(1921 / 6456)$ with higher prevalence in females than in males ( $42.4 \%$ versus $16.5 \%)$. The most common thyroid antibody positivity status was $\mathrm{TPOAb}^{+} \mathrm{TgAb}{ }^{+}$at $14.5 \%$ prevalence, followed by $\mathrm{TgAb}^{+}$alone at $12.4 \%$ and $\mathrm{TPOAb}^{+}$ alone at $2.9 \%$.

Table 1 summarizes the baseline characteristics of subjects grouped according to thyroid antibody status. There were significant differences between the four groups for age, gender, BMI, WC, SBP, FPG, TSH, TgAb, TPOAb, TG and HDL-C (all $P<0.001$ ). TC and LDL-C levels were not significantly different between the groups $\left(P=0.427\right.$ and $P=0.646$, respectively). Subjects with $\operatorname{TPOAb}^{+} \mathrm{TgAb}^{-}$were significantly older and had higher TgAb levels than control group subjects with negative antibodies (TPOAb-TgAb-) $(P<0.001)$. In the TPOAb-TgAb ${ }^{+}$group, BMI, WC, SBP, FPG and TG were found to be significantly lower than the control group $(P<0.001)$, while the percentage of women, TSH, TPOAb and HDL-C were significantly higher than the control group $(P<0.001)$. In the TPOAb ${ }^{+} \mathrm{TgAb}^{+}$group, age, $\mathrm{BMI}$, WC, SBP, FPG and TG were significantly lower than the control group, but the percentage of women, TSH, and HDL-C were observed to be significantly higher than the control group $(P<0.05)$.

\section{Associations between increasing serum lipid levels and $\operatorname{TgAb}$ positivity}

Table 2 presents the associations of serum lipid levels with TgAb positivity in the entire study population. After adjusting for age and gender in Model 1, the risk of $\operatorname{TgAb}$ positivity was positively associated with increasing levels of LDL-C. For every $1 \mathrm{mmol} / \mathrm{L}$ increase in LDL-C, the odds of having TgAb positivity increases by $8 \%(95 \% \mathrm{Cl} 1.00-1.16, P=0.044)$. In contrast, there were no significant associations between increasing TG, TC, or HDL-C levels and TgAb positivity $(P=0.413, P=0.402, P=0.125$, respectively). In Model 2, after further adjustment for BMI, WC, SBP and FPG, the positive association between LDL-C and TgAb positivity became more pronounced (OR 1.10, 95\% Cl 1.02-1.19, $P=0.014)$. Another finding includes that the risk of TgAb positivity was inversely associated with increasing levels of $\mathrm{HDL}-\mathrm{C}(\mathrm{OR} 0.80,95 \% \mathrm{Cl}$ 0.67-0.96, $P=0.014)$. There was no significant association between increasing TG or TC levels and TgAb positivity ( $P=0.846$ and $P=0.211$, respectively).

In the gender subgroup analyses, after full adjustment for age, BMI, WC, SBP and FPG, the relationships between increasing HDL-C (OR 0.74, 95\% $\mathrm{Cl} 0.60-0.91, P=0.005)$ or LDL-C $(\mathrm{OR} 1.15,95 \% \mathrm{Cl} 1.05-1.26, P=$ 0.004 ) levels and $\operatorname{TgAb}$ positivity were apparent only in female subjects (Table 2). There was no significant association between increasing lipid levels and TgAb positivity in male subjects (all $P>0.05$ ) (Table 2).

\section{Associations between increasing serum lipid levels and TPOAb positivity}

Associations between increasing serum lipid levels and TPOAb positivity were investigated in the same manner and is presented in Table 3 . In the entire study population, after adjustment for age and gender in Model 1, no significant association was found between any type of lipid parameter and TPOAb positivity 
(all $P>0.05$ ). Further adjustment for BMI, WC, SBP and FPG in Model 2, yielded similar results (all $P>$ $0.05)$.

In the gender subgroup analysis for women in Model 1, the risk of TPOAb positivity was positively correlated with increasing levels of LDL-C (OR 1.11, 95\% CI 1.01-1.24, $P=0.041)$. After full adjustment in Model 2, the trend became more significant between LDL-C levels and TPOAb positivity $(O R 1.14,95 \% \mathrm{Cl}$ 1.02-1.26, $P=0.016)$, no significant connection was observed between TG $(P=0.377)$, TC $(P=0.153)$ or HDL-C $(P=0.064)$ levels and TPOAb positivity (Table 3$)$. For men, there was no significant relationship between any type of lipid parameter and TPOAb positivity (all $P>0.05$ ) (Table 3 ).

\section{Discussion}

The present study focuses on the association between serum lipids and thyroid autoimmunity. We have shown the significant associations of increasing HDL-C and LDL-C levels with thyroid antibodies (TPOAb and TgAb) in the general population with normal TSH levels in a gender-dependent manner. Some literature has concentrated on the effects of thyroid autoantibodies on lipids [15]. However, few studies have explored the relationship between lipids and the positivity of thyroid autoantibodies. Especially, recent data have shown that serum lipids are novel risk factors for developing autoimmune diseases, such as systemic lupus erythematosus (SLE), celiac disease, idiopathic thrombocytopenic purpura (ITP) and Sjögren's disease $[8,16]$. Therefore, we aimed to evaluate whether similar associations can be observed in thyroid autoimmunity.

TSH is a pituitary gland hormone that plays a vital role in maintaining normal thyroid function. Abnormal levels of serum TSH can lead to dyslipidemia $[17,18]$, and it's association with serum lipid levels may be independent of thyroid hormone action[19]. Hence, our study was conducted in a normal TSH population. Interestingly, our findings suggested that TSH levels of the $\mathrm{TgAb}^{+}$groups were higher than the negative control group even though TSH is within the reference range. Similar results have been reported in the previous studies $[5,6,20]$ and may be explained by the fact that positivity for thyroid autoantibodies is associated with significantly higher risks of overt and subclinical hypothyroidism.

In the present study, we observed that lower concentrations of HDL-C were associated with a higher risk of TgAb positivity, which contrary to the result from an earlier study [15]. In this report, higher HDL-C levels were found in TPOAb-TgAb ${ }^{+}$when compared with TPOAb-TgAb-. The likely explanation for this is that the statistical analysis methods used are different. Zhang et al. [15] analyzed the relationships between lipids and thyroid autoantibody by analysis of variance, which was statistically less robust than the binary logistic regression from our study. Furthermore, we found no association between HDL-C levels and TPOAb positivity. Several studies support our finding [6,7,21-24] but conflicting evidence have also been reported. For example, TPOAb was found negatively correlated with HDL-C levels in Iranian and Turkish studies by Tamer et al. and Mazaheri et al., respectively [3,4]. The reason for the discrepancy may be due to differences in the study design. These case-control studies had predominantly patients with HT who have higher titers of TPOAb and much higher risk of overt and subclinical hypothyroidism in comparison 
to the general population. In contrast, a domestic study found that TPOAb was positively correlated to HDL-C levels [5], which is supported by the Copenhagen City Heart Study by Madsen et al. [8]. The Copenhagen Study reported U-shaped associations between HDL-C and autoimmune diseases, that is, high concentrations of HDL-C ( $>77 \mathrm{mg} / \mathrm{dL}$ ) is also associated with higher risk of autoimmune diseases. It seems to differ from the traditional perception of HDL-C as the "good cholesterol". Possible explanations include: (i) the presence of residual confounding factors such as cancer, infection and other influence factors affected the results [25]; (ii) the presence of genetic variants [26] changed composition and function of HDL particles; and (iii) individuals who have high HDL-C (> $60 \mathrm{mg} / \mathrm{dL})$ and very high HDL$\mathrm{C}(>100 \mathrm{mg} / \mathrm{dL})$ concentrations are often grouped with participants with moderate concentrations. As a result of the above factors, researchers may not be able to observe this U-shaped relationship between HDL-C and autoimmune diseases [27,28].

Our data also suggested that increasing LDL-C levels were positively associated with TgAb and TPOAb positivity, which is supported by previous studies $[5,7,24]$. However, we did not find any association between TC levels and TgAb or TPOAb positivity, even though roughly seventy percent of serum TC is composed of LDL-C and the level of TC is usually parallel to that of LDL-C [5,29]. This lack of association observed in our study is inconsistent with previous reports. Kang et al. and Srivastava et al. reported that serum TC and LDL-C were positively correlated to TPOAb [5,7]. In another study, serum TC and LDL-C were found positively correlated to $\operatorname{TgAb}$ [24]. Since TC is the aggregate of cholesterol contained in various lipoproteins in the blood, including LDL-C, HDL-C, extremely low-density lipoprotein (VLDL), lipoprotein (a), and others, it is not ruled out that the association between TC and thyroid autoantibody may be affected by other cholesterol composition. Therefore, caution should be exercised when interpreting the results from the relationship of TC and LDL-C with the thyroid autoantibodies.

Moreover, we did not observe any association between TG and thyroid autoantibodies, which is consistent with several reports $[4,6,21]$. However, TG has also been observed to be positively correlated with TgAb and/or TPOAb in some studies $[3,7,22]$. These differences in findings could be due to geographical location, ethnicity, genetics, thyroid autoantibody cutoff values, sample size and statistical analyses methods.

Unexpectedly, we detected that the relationships between the serum lipids and thyroid autoantibody positivity were significant only in women. The disparate results from gender could be related to the immune system exhibiting sexual dimorphism in humans [30]. The unique effect of estrogen on the immune system may determine the susceptibility of women to autoimmune diseases and the greater immune response in women than in men [31].

Three main questions arise based on our study: First, what are the underlying mechanisms of the associations between serum lipids and thyroid autoantibodies? Evidence indicates that toll-like receptors (TLRs) play an essential role in the pathogenesis of AITD, HDL and LDL can inhibit and activate TLRs thus affect the progress of Graves' disease (GD) or HT, respectively [32-35]. In addition, reactive oxygen species (ROS) are necessary for thyroid hormone synthesis in physiological conditions, but excessive 
ROS may result in thyrocytes apoptosis and necrosis. HDL and LDL are capable of modulating and stimulating oxidative stress further involvement in the development of AITD, respectively [36-38]. Further research is required in this field. Second, would management of blood lipids be beneficial to the patients at high risk of thyroid autoimmunity, given that lower serum HDL-C level or higher serum LDL-C level is associated with higher risk of this disease? Third, whether management of blood lipids could specifically prevent the onset of AITD in susceptible populations or decrease thyroid autoantibody titer? These questions of great clinical significance remain to be explored in the future.

To the best of our knowledge, this is the first report evaluating the issue of the association of serum lipids with thyroid autoantibody in the general population from a serum lipid perspective, and we unexpectedly identified that these relationships differed with gender. Moreover, in comparison to other reports, our study had a larger sample size and stronger statistical methods, which made the results more reliable. However, several limitations of this study should also be considered. First, due to the cross-sectional design of this study, the direction of any causal relationship could not be established. Second, this study was conducted in a single center, which suggests that the results may be inadequate to generalize to the broader population or even to different races. Finally, due to the lack of information on medication, smoking, alcohol consumption, and other concomitant autoimmune diseases, the results may also be affected by some unknown confounding factors.

In conclusion, we have shown the associations of increasing HDL-C and LDL-C levels with thyroid autoantibody positivity in the general population with normal TSH levels in a gender-dependent manner. This study highlights that lipid profiles may be new predictors of thyroid autoimmunity even when TSH is within the normal reference range. However, further validation is required in future prospective studies.

\section{Declarations}

\section{Acknowledgments}

First, we would like to thank all the participants of this study. Second, we would like to acknowledge Yongze Li, MD and PhD for his contribution in the statistical analyses. Third, we would like to thank English native speaker Di Wu, MBBS for editing the English language of the manuscript.

Funding This work was supported by the National Natural and Science Foundation of China (grant number: 81870538).

Conflicts of interest The authors declare that they have no conflict of interest.

\section{Availability of data and material}

Not applicable.

\section{Code availability}


Not applicable.

Authors' contributions H.G. and W.W. contributed to the study conception and design. Material preparation and data collection and analysis were performed by J.L., Z.W., H.L. and J.F. and F.Q. The first draft of the manuscript was written by J.L. and Z.W. All authors commented on the previous versions of the manuscript. All authors read and approved the final manuscript.

Ethics approval This retrospective study was approved by the Medical Ethics Committee of The First Hospital of China Medical University. Informed consent waiver was obtained to use deidentified data.

\section{Consent to participate}

Not applicable.

\section{Consent for publication}

Not applicable.

\section{References}

1. Li, Y., Teng, D., Ba, J., Chen, B., Du, J., He, L., Lai, X., Teng, X., Shi, X., Li, Y., Chi, H., Liao, E., Liu, C., Liu, L., Qin, G., Qin, Y., Quan, H., Shi, B., Sun, H., Tang, X., Tong, N., Wang, G., Zhang, J.A., Wang, Y., Xue, Y., Yan, L., Yang, J., Yang, L., Yao, Y., Ye, Z., Zhang, Q., Zhang, L., Zhu, J., Zhu, M., Ning, G., Mu, Y., Zhao, J., Shan, Z., Teng, W.: Efficacy and Safety of Long-Term Universal Salt lodization on Thyroid Disorders: Epidemiological Evidence from 31 Provinces of Mainland China. Thyroid 30(4), 568-579 (2020). doi:10.1089/thy.2019.0067

2. Fowler, P.S., Swale, J.: Premyxoedema and coronary-artery disease. Lancet 1(7499), 1077-1079 (1967). doi: 10.1016/s0140-6736(67)92650-5

3. Tamer, G., Mert, M., Tamer, I., Mesci, B., Kilic, D., Arik, S.: Effects of thyroid autoimmunity on abdominal obesity and hyperlipidaemia. Endokrynol Pol 62(5), 421-428 (2011).

4. Mazaheri, T., Sharifi, F., Kamali, K.: Insulin resistance in hypothyroid patients under Levothyroxine therapy: a comparison between those with and without thyroid autoimmunity. J Diabetes Metab Disord 13(1), 103 (2014). doi:10.1186/s40200-014-0103-4

5. Kang, D., Yin, Q., Yan, X., Song, H., Gao, G., Liang, J., Zhao, J.: Serum cholesterol levels in middle-aged euthyroid subjects with positive thyroid peroxidase antibodies. Int J Clin Exp Med 8(11), 2162321628 (2015).

6. Chen, Y., Zhu, C., Chen, Y., Wang, N., Li, Q., Han, B., Zhao, L., Chen, C., Zhai, H., Zhang, L., Lu, Y.: Are Thyroid Autoimmune Diseases Associated with Cardiometabolic Risks in a Population with Normal Thyroid-Stimulating Hormone? Mediators Inflamm 2018, 1856137 (2018). doi:10.1155/2018/1856137 
7. Srivastava, V.K., Singh, H.: Association of thyroid peroxidase antibody and dyslipidemia in subclinical hypothyroidism. J Family Med Prim Care 6(1), 63-68 (2017). doi:10.4103/2249-4863.214958

8. M, M.C., Anette, V., G, N.B.: Low HDL Cholesterol and High Risk of Autoimmune Disease: Two Population-Based Cohort Studies Including 117341 Individuals. Clin Chem 65(5), 644-652 (2019). doi: 10.1373/clinchem.2018.299636

9. Fessler, M.B.: Regulation of Adaptive Immunity in Health and Disease by Cholesterol Metabolism. Curr Allergy Asthma Rep 15(8), 48 (2015). doi:10.1007/s11882-015-0548-7

10. Ito, A., Hong, C., Oka, K., Salazar, J.V., Diehl, C., Witztum, J.L., Diaz, M., Castrillo, A., Bensinger, S.J., Chan, L., Tontonoz, P.: Cholesterol Accumulation in CD11c Immune Cells Is a Causal and Targetable Factor in Autoimmune Disease. Immunity 45(6), 1311-1326 (2016).

doi:10.1016/j.immuni.2016.11.008

11. Stein, J.D., Childers, D., Gupta, S., Talwar, N., Nan, B., Lee, B.J., Smith, T.J., Douglas, R.: Risk factors for developing thyroid-associated ophthalmopathy among individuals with Graves disease. JAMA ophthalmology 133(3), 290-296 (2015). doi:10.1001/jamaophthalmol.2014.5103

12. Gullu, S., Emral, R., Bastemir, M., Parkes, A.B., Lazarus, J.H.: In vivo and in vitro effects of statins on lymphocytes in patients with Hashimoto's thyroiditis. Eur J Endocrinol 153(1), 41-48 (2005). doi:10.1530/eje.1.01941

13. Wu, X., Schott, M., Liu, C., Qian, C., Mao, X., Xu, K., Jiang, J., Xu, Y., Shen, M., Papewalis, C., Scherbaum, W.A., Liu, C.: Statins decrease the aberrant HLA-DR expression on thyrocytes from patients with Hashimoto's thyroiditis. Horm Metab Res 40(12), 838-841 (2008). doi:10.1055/s-20081081500

14. Krysiak, R., Szkróbka, W., Okopień, B.: The Effect of Hypolipidemic Agents on Thyroid Autoimmunity in Women with Hashimoto's Thyroiditis Treated with Levothyroxine and Selenomethionine. Exp Clin Endocrinol Diabetes 126(5), 321-326 (2018). doi:10.1055/s-0043-120342

15. Zhang, J., Gao, Y., Li, Y., Teng, D., Li, J.: The Presence of Serum TgAb Suggests Lower Risks for Glucose and Lipid Metabolic Disorders in Euthyroid General Population From a National Survey. Front Endocrinol (Lausanne) 11 (2020). doi: 10.3389/fendo.2020.00139

16. Tselios, K., Koumaras, C., Gladman, D.D., Urowitz, M.B.: Dyslipidemia in systemic lupus erythematosus: just another comorbidity? Semin Arthritis Rheum 45(5), 604-610 (2016). doi:10.1016/j.semarthrit.2015.10.010

17. Langén, V.L., Niiranen, T.J., Puukka, P., Sundvall, J., Jula, A.M.: Association of thyroid-stimulating hormone with lipid concentrations: an 11-year longitudinal study. Clin Endocrinol (Oxf) 86(1), 120127 (2017). doi:10.1111/cen.13151

18. Santos-Palacios, S., Brugos-Larumbe, A., Guillén-Grima, F., Galofré, J.C.: A cross-sectional study of the association between circulating TSH level and lipid profile in a large Spanish population. Clin Endocrinol (Oxf) 79(6), 874-881 (2013). doi:10.1111/cen.12216

19. Xu, C., Yang, X., Liu, W., Yuan, H., Yu, C., Gao, L., Zhao, J.: Thyroid stimulating hormone, independent of thyroid hormone, can elevate the serum total cholesterol level in patients with coronary heart 
disease: a cross-sectional design. Nutr Metab (Lond) 9(1), 44 (2012). doi:10.1186/1743-7075-9-44

20. Teng, D., Yang, W., Shi, X., Li, Y., Ba, J., Chen, B., Du, J., He, L., Lai, X., Li, Y., Chi, H., Liao, E., Liu, C., Liu, L., Qin, G., Qin, Y., Quan, H., Shi, B., Sun, H., Tang, X., Tong, N., Wang, G., Zhang, J.A., Wang, Y., Xue, Y., Yan, L., Yang, J., Yang, L., Yao, Y., Ye, Z., Zhang, Q., Zhang, L., Zhu, J., Zhu, M., Shan, Z., Teng, W.: An inverse relationship between iodine intake and thyroid antibodies: a national cross-sectional survey in mainland China. Thyroid 30(11), 1656-1665 (2020). doi:10.1089/thy.2020.0037

21. Wells, B.J., Hueston, W.J.: Are thyroid peroxidase antibodies associated with cardiovascular disease risk in patients with subclinical hypothyroidism? Clin Endocrinol (Oxf) 62(5), 580-584 (2005). doi: 10.1111/j.1365-2265.2005.02262.x

22. Topaloglu, O., Gokay, F., Kucukler, K., Burnik, F.S., Mete, T., Yavuz, H.C., Berker, D., Guler, S.: Is autoimmune thyroiditis a risk factor for early atherosclerosis in premenopausal women even if in euthyroid status? Endocrine 44(1), 145-151 (2013). doi:10.1007/s12020-012-9842-5

23. Xiaodong, C., Jie, S.: The study of the level of blood fat and the relationship between the level of thyroid autoimmune antibody in hyperthyroidism patients. Med $\mathrm{J}$ of Communications 30(2), 117-120 (2016).

24. Chai, L., Liu, X., Ma, Y., Zhang, L., Zheng, L.: The study of the level of blood lipid and the relationship between thyroid hormone and autoantibody in AITD patients. Henan Med Res 26(1), 25-28 (2017). doi:10.3969/j.issn.1004-437X.2017.01.009

25. Hamer, M., O’Donovan, G., Stamatakis, E.: High-Density Lipoprotein Cholesterol and Mortality: Too Much of a Good Thing? Arterioscler Thromb Vasc Biol 38(3), 669-672 (2018). doi: 10.1161/ATVBAHA.117.310587

26. Motazacker, M.M., Peter, J., Treskes, M., Shoulders, C.C., Kuivenhoven, J.A., Hovingh, G.K.: Evidence of a polygenic origin of extreme high-density lipoprotein cholesterol levels. Arterioscler Thromb Vasc Biol 33(7), 1521-1528 (2013). doi:10.1161/ATVBAHA.113.301505

27. Di Angelantonio, E., Sarwar, N., Perry, P., Kaptoge, S., Ray, K.K., Thompson, A., Wood, A.M., Lewington, S., Sattar, N., Packard, C.J., Collins, R., Thompson, S.G., Danesh, J.: Major lipids, apolipoproteins, and risk of vascular disease. JAMA 302(18), 1993-2000 (2009). doi:10.1001/jama.2009.1619

28. Gordon, D.J., Probstfield, J.L., Garrison, R.J., Neaton, J.D., Castelli, W.P., Knoke, J.D., Jacobs, D.R., J., Bangdiwala, S., Tyroler, H.A.: High-density lipoprotein cholesterol and cardiovascular disease. Four prospective American studies. Circulation 79(1), 8-15 (1989). doi:10.1161/01.cir.79.1.8

29. Expert Panel on Detection, Evaluation, and Treatment of High Blood Cholesterol in Adults, Executive Summary of The Third Report of The National Cholesterol Education Program (NCEP) Expert Panel on Detection, Evaluation, And Treatment of High Blood Cholesterol In Adults (Adult Treatment Panel III). JAMA 285(19), 2486-2497 (2001).

30. Amur, S., Parekh, A., Mummaneni, P.: Sex differences and genomics in autoimmune diseases. J Autoimmun 38(2-3), J254-J265 (2012). doi:10.1016/j.jaut.2011.12.001

31. Fish, E.N.: The X-files in immunity: sex-based differences predispose immune responses. Nat Rev Immunol 8(9), 737-744 (2008). doi:10.1038/nri2394 
32. Miller, Y.I., Viriyakosol, S., Binder, C.J., Feramisco, J.R., Kirkland, T.N., Witztum, J.L.: Minimally modified LDL binds to CD14, induces macrophage spreading via TLR4/MD-2, and inhibits phagocytosis of apoptotic cells. J Biol Chem 278(3), 1561-1568 (2003). doi:

10.1074/jbc.M209634200

33. Dvornikova, K.A., Bystrova, E.Y., Platonova, O.N., Churilov, L.P.: Polymorphism of toll-like receptor genes and autoimmune endocrine diseases. Autoimmun Rev 19(4), 102496 (2020). doi:10.1016/j.autrev.2020.102496

34. Wu, B.J., Ong, K.L., Shrestha, S., Chen, K., Tabet, F., Barter, P.J., Rye, K.-A.: Inhibition of arthritis in the Lewis rat by apolipoprotein A-I and reconstituted high-density lipoproteins. Arterioscler Thromb Vasc Biol 34(3), 543-551 (2014). doi:10.1161/ATVBAHA.113.302832

35. Suzuki, M., Pritchard, D.K., Becker, L., Hoofnagle, A.N., Tanimura, N., Bammler, T.K., Beyer, R.P., Bumgarner, R., Vaisar, T., de Beer, M.C., de Beer, F.C., Miyake, K., Oram, J.F., Heinecke, J.W.: Highdensity lipoprotein suppresses the type I interferon response, a family of potent antiviral immunoregulators, in macrophages challenged with lipopolysaccharide. Circulation 122(19), 19191927 (2010). doi:10.1161/CIRCULATIONAHA.110.961193

36. Ates, I., Yilmaz, F.M., Altay, M., Yilmaz, N., Berker, D., Güler, S.: The relationship between oxidative stress and autoimmunity in Hashimoto's thyroiditis. Eur J Endocrinol 173(6), 791-799 (2015). doi:10.1530/EJE-15-0617

37. Hermida, N., Balligand, J.-L.: Low-density lipoprotein-cholesterol-induced endothelial dysfunction and oxidative stress: the role of statins. Antioxid Redox Signal 20(8), 1216-1237 (2014). doi:10.1089/ars.2013.5537

38. Xepapadaki, E., Zvintzou, E., Kalogeropoulou, C., Filou, S., Kypreos, K.E.: The Antioxidant Function of HDL in Atherosclerosis. Angiology 71(2), 112-121 (2020). doi:10.1177/0003319719854609

\section{Tables}

Table 1 Baseline characteristics of subjects according to thyroid antibody status 


\begin{tabular}{|c|c|c|c|c|c|}
\hline & $\mathrm{TPOAb}^{+} \mathrm{TgAb}^{-}$ & TPOAb-TgAb ${ }^{+}$ & $\mathrm{TPOAb}^{+} \mathrm{TgAb}^{+}$ & TPOAb-TgAb- & $\begin{array}{l}P \text { - } \\
\text { value }\end{array}$ \\
\hline Number (\%) & $184(2.9)$ & $800(12.4)$ & $937(14.5)$ & $4535(70.2)$ & - \\
\hline Men (\%) & $104(56.5)$ & $226(28.2)^{b}$ & $191(20.4)^{b}$ & $2637(58.1)$ & $<0.001$ \\
\hline Age (year) & $51.24 \pm 10.69^{b}$ & $47.18 \pm 11.46$ & $46.70 \pm 10.90^{a}$ & $\begin{array}{l}47.77 \pm \\
11.16\end{array}$ & $<0.001$ \\
\hline $\mathrm{BMI}\left(\mathrm{Kg} / \mathrm{m}^{2}\right)$ & $24.97 \pm 3.26$ & $24.39 \pm 3.27^{b}$ & $24.13 \pm 3.39^{b}$ & $25.23 \pm 3.56$ & $<0.001$ \\
\hline WC (cm) & $82.72 \pm 10.38$ & $80.04 \pm 10.50^{b}$ & $78.46 \pm 9.92^{b}$ & $\begin{array}{l}83.96 \pm \\
10.83\end{array}$ & $<0.001$ \\
\hline $\begin{array}{l}\text { SBP } \\
(\mathrm{mmHg})\end{array}$ & $130.08 \pm 21.01$ & $\begin{array}{l}126.02 \pm 19.79 \\
b\end{array}$ & $124.18 \pm 18.42^{b}$ & $\begin{array}{l}128.79 \pm \\
19.05\end{array}$ & $<0.001$ \\
\hline $\begin{array}{l}\text { FPG } \\
(\mathrm{mmol} / \mathrm{L})\end{array}$ & $5.23(4.89-5.67)$ & $\begin{array}{l}5.08(4.79-5.43) \\
b\end{array}$ & $5.09(4.84-5.42)^{b}$ & $\begin{array}{l}5.16(4.83- \\
5.59)\end{array}$ & $<0.001$ \\
\hline TSH (mlU/L) & $1.66(1.14-2.36)$ & $\begin{array}{l}1.68(1.20-2.43) \\
b\end{array}$ & $2.11(1.43-3.12)^{b}$ & $\begin{array}{l}1.55(1.12- \\
2.15)\end{array}$ & $<0.001$ \\
\hline $\begin{array}{l}\text { TPOAb } \\
(\mathrm{IU} / \mathrm{ml})\end{array}$ & $\begin{array}{l}17.41(8.91- \\
48.15)^{b}\end{array}$ & $\begin{array}{l}0.52(0.24-1.16) \\
b\end{array}$ & $\begin{array}{l}158.26(42.96- \\
519.18)^{b}\end{array}$ & $\begin{array}{l}0.29(0.12- \\
0.58)\end{array}$ & $<0.001$ \\
\hline $\operatorname{TgAb}(\mathrm{IU} / \mathrm{ml})$ & $\begin{array}{l}2.21(1.52-2.92) \\
\mathrm{b}\end{array}$ & $\begin{array}{l}13.26(6.05- \\
51.11)^{b}\end{array}$ & $\begin{array}{l}45.23(16.58- \\
171.73)^{b}\end{array}$ & $\begin{array}{l}1.46(1.04- \\
2.03)\end{array}$ & $<0.001$ \\
\hline $\begin{array}{l}\text { TG } \\
(\mathrm{mmol} / \mathrm{L})\end{array}$ & $1.23(0.88-1.82)$ & $\begin{array}{l}1.18(0.81-1.72) \\
b\end{array}$ & $1.12(0.80-1.70)^{b}$ & $\begin{array}{l}1.34(0.89- \\
2.07)\end{array}$ & $<0.001$ \\
\hline TC (mmol/L) & $4.98 \pm 0.90$ & $4.98 \pm 0.95$ & $4.98 \pm 0.89$ & $4.94 \pm 0.90$ & 0.427 \\
\hline $\begin{array}{l}\mathrm{HDL}-\mathrm{C} \\
(\mathrm{mmol} / \mathrm{L})\end{array}$ & $1.35 \pm 0.37$ & $1.39 \pm 0.37^{b}$ & $1.43 \pm 0.37^{b}$ & $1.32 \pm 0.37$ & $<0.001$ \\
\hline $\begin{array}{l}\text { LDL-C } \\
(\mathrm{mmol} / \mathrm{L})\end{array}$ & $3.16 \pm 0.77$ & $3.12 \pm 0.82$ & $3.11 \pm 0.77$ & $3.10 \pm 0.80$ & 0.646 \\
\hline
\end{tabular}

$B M I$ body mass index, $W C$ waist circumference, $S B P$ systolic blood pressure, $F P G$ fasting plasma glucose, $T S H$ thyroid-stimulating hormone, TPOAb thyroid peroxidase antibody, TgAb thyroglobulin antibody, TG triglyceride, $T C$ total cholesterol, $H D L-C$ high-density lipoprotein cholesterol, $L D L-C$ low-density lipoprotein cholesterol

The data are presented as $\mathrm{n}(\%)$, median (first quartile-third quartile) or mean $\pm \mathrm{SD}$

${ }^{a} P<0.05$ compared to TPOAb-TgAb- group

${ }^{\mathrm{b}} P<0.01$ compared to TPOAb-TgAb- group 
Table 2 Association of lipid levels with $\mathrm{TgAb}$ positivity

\begin{tabular}{|llll|}
\hline Each 1-mmol/L increase & Case/number $(\%)$ & Model 1 & Model 2 \\
\hline Total & & & \\
\hline TG & $1739 / 6456(26.9)$ & $0.98(0.93-1.03)$ & $1.01(0.95-1.06)$ \\
\hline TC & $1739 / 6456(26.9)$ & $1.03(0.96-1.10)$ & $1.04(0.98-1.11)$ \\
\hline HDL-C & $1739 / 6456(26.9)$ & $0.88(0.74-1.04)$ & $0.80(0.67-0.96)^{a}$ \\
\hline LDL-C & $1739 / 6456(26.9)$ & $1.08(1.00-1.16)^{a}$ & $1.10(1.02-1.19)^{a}$ \\
\hline TG & & & \\
\hline TC & $1322 / 3298(40.1)$ & $1.01(0.94-1.08)$ & $1.04(0.97-1.13)$ \\
\hline HDL-C & $1322 / 3298(40.1)$ & $1.06(0.98-1.15)$ & $1.07(0.99-1.16)$ \\
\hline LDL-C & $1322 / 3298(40.1)$ & $0.82(0.67-1.00)^{a}$ & $0.74(0.60-0.91)^{a}$ \\
\hline Men & $1322 / 3298(40.1)$ & $1.13(1.03-1.24)^{a}$ & $1.15(1.05-1.26)^{a}$ \\
\hline TG & & & \\
\hline TC & $417 / 3158(13.2)$ & $0.96(0.89-1.03)$ & $0.97(0.90-1.05)$ \\
\hline HDL-C & $417 / 3158(13.2)$ & $0.99(0.88-1.11)$ & $1.00(0.89-1.12)$ \\
\hline LDL-C & $417 / 3158(13.2)$ & $1.03(0.75-1.42)$ & $0.96(0.69-1.35)$ \\
\hline & $417 / 3158(13.2)$ & $1.02(0.89-1.16)$ & $1.02(0.90-1.17)$ \\
\hline
\end{tabular}

$T G$ triglyceride, $T C$ total cholesterol, $H D L-C$ high-density lipoprotein cholesterol, $L D L-C$ low-density lipoprotein cholesterol

${ }^{\mathrm{a}} P<0.05$

Model 1: adjusted for age and/or gender

Model 2: further adjusted for BMI, WC, SBP and FPG

Table 3 Association of lipid levels with TPOAb positivity 


\begin{tabular}{|llll|}
\hline Each 1-mmol/L increase & Case/number (\%) & Model 1 & Model 2 \\
\hline Total & & & \\
\hline TG & $1123 / 6456(17.4)$ & $0.95(0.90-1.01)$ & $0.98(0.92-1.05)$ \\
\hline TC & $1123 / 6456(17.4)$ & $1.01(0.94-1.09)$ & $1.03(0.96-1.11)$ \\
\hline HDL-C & $1123 / 6456(17.4)$ & $0.99(0.82-1.20)$ & $0.89(0.73-1.09)$ \\
\hline LDL-C & $1123 / 6456(17.4)$ & $1.05(0.96-1.14)$ & $1.08(0.99-1.17)$ \\
\hline Tomen & & & \\
\hline TC & $828 / 3298(25.1)$ & $1.01(0.93-1.09)$ & $1.04(0.96-1.13)$ \\
\hline HDL-C & $828 / 3298(25.1)$ & $1.06(0.97-1.16)$ & $1.07(0.98-1.17)$ \\
\hline LDL-C & $828 / 3298(25.1)$ & $0.89(0.71-1.11)$ & $0.80(0.63-1.01)$ \\
\hline Men & $828 / 3298(25.1)$ & $1.11(1.01-1.24)^{a}$ & $1.14(1.02-1.26)^{a}$ \\
\hline TG & & & \\
\hline TC & $295 / 3158(9.3)$ & $0.91(0.82-1.00)^{a}$ & $0.93(0.84-1.03)$ \\
\hline HDL-C & $295 / 3158(9.3)$ & $0.97(0.85-1.11)$ & $0.98(0.86-1.13)$ \\
\hline LDL-C & $295 / 3158(9.3)$ & $1.28(0.90-1.80)$ & $1.18(0.82-1.71)$ \\
\hline & $295 / 3158(9.3)$ & $0.98(0.84-1.14)$ & $0.99(0.85-1.16)$ \\
\hline
\end{tabular}

$T G$ triglyceride, $T C$ total cholesterol, $H D L-C$ high-density lipoprotein cholesterol, $L D L-C$ low-density lipoprotein cholesterol

a $P<0.05$

Model 1: adjusted for age and/or gender

Model 2: further adjusted for BMI, WC, SBP and FPG 